\title{
Fluidic resistance control enables high-throughput establishment of mixed-species biofilms
}

\author{
Mads Frederik Hansen', Anders Meyer Torp², Jonas Stenløkke Madsen', Henriette Lyng Røder ${ }^{\star}, 1$ \& Mette Burmølle*,1
}

\section{ABSTRACT}

Bacteria often live in communities of mixed species embedded in a selfproduced extracellular matrix of polysaccharides, proteins and DNA, termed biofilms. The BioFlux microfluidic flow system is useful for studying biofilm formation in different media under flow. However, analyzing the architecture and maturation of biofilms under flow requires a proper seeding, which can prove difficult when working with bacteria of different sizes, motile bacteria or aiming for a high number of replicates. Here we developed an efficient protocol that exploits viscosity tuning and seeding indicator dyes to improve seeding and allow for high-throughput examination and visualization of consistent mono- and mixedspecies biofilm developments under flow.

\section{METHOD SUMMARY}

An improved protocol for seeding bacteria in the BioFlux microfluidic flow system was developed using glycerol and Nigrosin to increase the viscosity and stain the media, respectively. This new protocol does not alter biofilm development and allows consistent mixedspecies biofilm formation in a copious number of replicates.

\section{KEYWORDS:}

biofilm analysis $\cdot$ biofilm device $\cdot$ biofilm formation - biofilm seeding $\cdot$ BioFlux $\mathrm{CLSM} \cdot$ microfluidic device $\cdot$ microfluidic flow systems $\cdot$ mixed species biofilm . shear stress

'Section of Microbiology, Universitetsparken 15, Department of Biology, Faculty of Science, University of Copenhagen, 2100 Copenhagen, Denmark; ${ }^{2}$ Research Group for Gut, Microbes \& Health, Kemitorvet, National Food Institute, Technical University of Denmark, 2800 Kgs. Lyngby, Denmark; "Author for correspondence: h.lyng.roeder@bio.ku.dk; **Author for correspondence:burmolle@bio.ku.dk

BioTechniques 66: 235-239 (May 2019) 10.2144/ btn-2018-0150
Bacterial biofilms are predominant in a wide range of habitats where cells form dense, structured and often mixed species communities encased in a matrix of extracellular polymeric substance (EPS). The matrix allows cells to cling to a surface or together as free-floating aggregates, detains extracellular compounds and enzymes and provides a protected environment enabling bacteria to persist for generations in otherwise hostile surroundings [1-3]. Biofilm formation is also of special interest since it promotes increased tolerance towards antimicrobials and is implicated in a variety of chronic infections [4].

Although the level of shear is known to affect biofilm formation [3,5], and the changed composition and spatial organization along flow channels has been found to affect antibiotic tolerance [6], studies of biofilms under flow are not as frequent as their static counterparts, as highthroughput systems have been lacking [7]. The importance of dynamic conditions was also noticeable in a collection of Pseudomonas aeruginosa mutants, generated by random mini-Tn5 mutations, as genes involved in biofilm formation, otherwise overlooked under static conditions, were identified [8].

The BioFlux microfluidic flow system (Fluxion Biosciences) has been developed in order to study biofilm formation in continuous flow over a broad range of controlled fluid shear, approximate natural biofilms known from, for example, medical devices or some human infections, while allowing simultaneous studies of up to 96 replicates. Biofilms established in the BioFlux system can be examined continuously with optical microscopy or confocal laser scanning microscopy (CLSM), enabling monitoring of attachment, maturation and potential dispersal under flow, while requiring minimal volumes of media compared with other continuous flow systems [7]. The small volume applied compared with other systems such as drip flow reactors makes it suitable for screening of biofilm inhibitory agents under flow [9]. Precise seeding is, however, required in order to avoid time waste and running cost of unsuccessful replicates. Seeding is performed by reversing the direction of the fluidic flow and allowing the inoculum to enter the viewing chamber from the outlet well, without contaminating the upstream channel and inlet well (Figure 1), which can prove challenging without hands-on experience. Moreover, recent research focuses on interspecies interactions in multispecies biofilms and the competition, cooperation, synergy and new microniches they promote [10], imposing further challenges on the model systems applied. Difficulties when seeding mixed bacterial communities efficiently and precisely are currently a significant constraint of the BioFlux system, which limits the reproducibility of highthroughput experiments. The swimming speed of bacterial cells affects settling dynamics [11], and motility also enables the cells to settle at unintended positions and inconsistently between channels. In addition, inadvertent residual flow during the attachment period, and different settling rates among bacterial cells of different sizes in the inoculum (both in the well and within the flow channel), cause difficulties when using this system to obtain consistent growth, especially for mixed-species biofilms. We here present an improved protocol for seeding the BioFlux system, designed to circumvent the challenges experienced in analysis of mixed-species cultures. By using glycerol to increase the viscosity of the seeding media and Nigrosin stain to visualize the inoculum, we enhanced throughput and producibility of bacterial seeding in the BioFlux microfluidic flow system. 

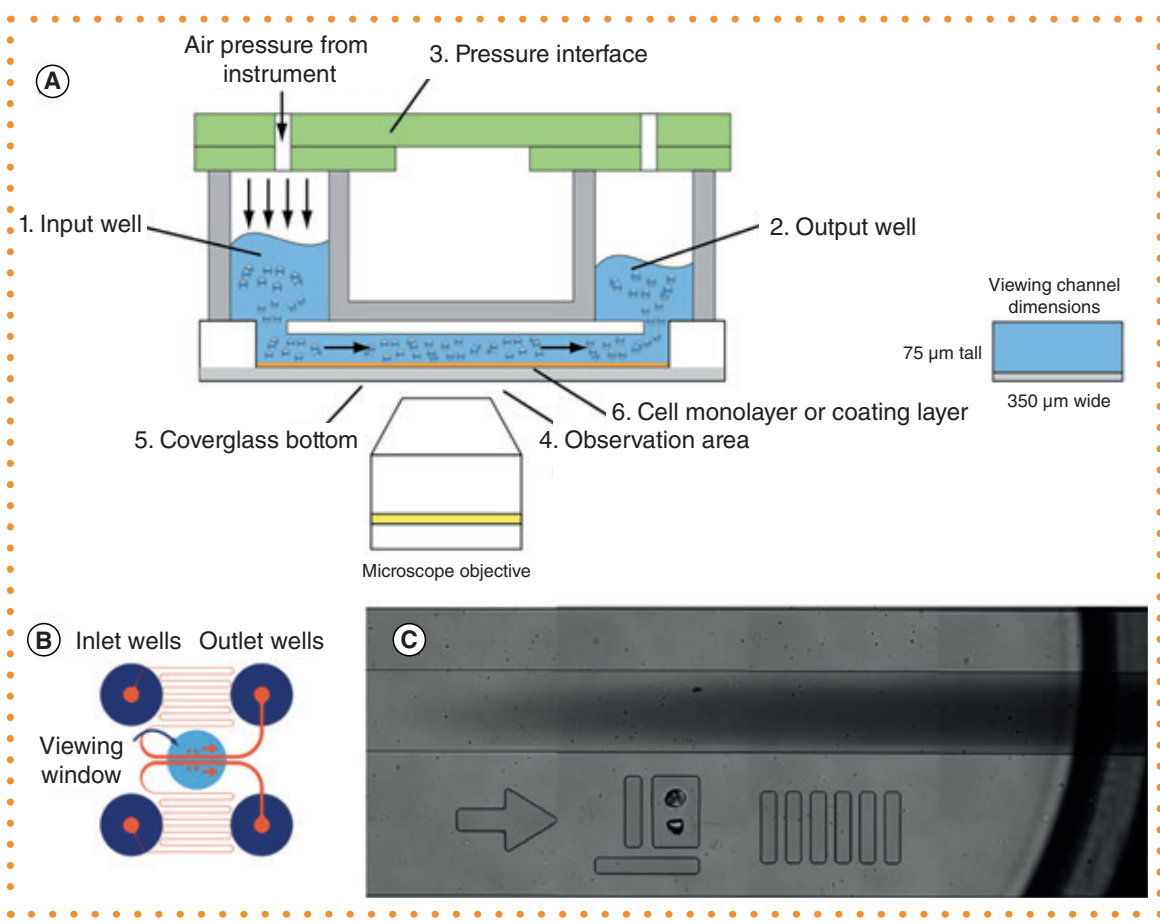

Figure 1. Schematic diagram of the BioFlux microfluidic flow system channel and example of seeding with Nigrosin at high viscosity. (A \& B) Each channel has an inlet well containing fresh medium, which is pushed through the channel and viewing chamber (containing the biofilm) and ends as spent medium in the outlet well, due to pneumatic pressure delivered to the top of the inlet well. Seeding of this system is performed by pushing the bacterial inoculum from the outlet well to the viewing chamber by well-coordinated reverse flow. (C) Staining of the bacterial inoculum (dark) enables visualization at low magnification.

- According to Stokes' law, settling velocity varies greatly with particle size and is related to fluid viscosity (Eq. 1).

$$
\mathrm{v}=\frac{2}{9} \frac{\left(\mathrm{p}_{\mathrm{p}}-\mathrm{p}_{\mathrm{f}}\right)}{\mathrm{m}} \mathrm{gR} \mathrm{R}^{2}
$$

Where $v$ is the settling velocity $(\mathrm{m} / \mathrm{s}), \mathrm{R}$ is the radius of the particle $(\mathrm{m}), \mu$ is the dynamic viscosity $(\mathrm{kg} / \mathrm{m} * \mathrm{~s}), \mathrm{g}$ is the gravitational acceleration $\left(\mathrm{m} / \mathrm{s}^{2}\right), \mathrm{p}_{\mathrm{p}}$ is the mass density of the particles and $p_{f}$ is the mass density of the fluid $\left(\mathrm{kg} / \mathrm{m}^{3}\right)$.

Increasing the medium viscosity with glycerol reduces gravity-induced flow and equalizes the settling time and simultaneously decreases unwanted flow during the attachment period. As bacteria are small and seeding is often performed with a few cells, they can be difficult to observe via microscopy at low magnification. Staining the inoculum provides a simple way for the operator to verify that the inoculum has been introduced in the viewing area quickly and easily, without reverting from low to high magnification microscopy (Figure 1C). This ensures correct bacterial positioning and improves the consistency of seeding, thus also the consistency of biofilm formation, and enables controlled high-throughput biofilm growth in this flow system.

A doubling in viscosity can be achieved at $20 \%$ glycerol concentration [12,13], which will lead to a halving of both settling velocity and residual flows due to gravity and surface tension effects. The same pressure driving flow will lead to lower flow speeds in the channel and result in better control of the position of the inoculum front. Longer settling times will lead to superior species mixing during inoculum introduction.

In this study we used fluorescently tagged strains of Pseudomonas putida, Paenibacillus amylolyticus and a wrinkled variant of Xanthomonas retroflexus in order to demonstrate the improvements provided by this new protocol. These bacteria represent a great model, due to their ability to grow under the same conditions, and exemplify the challenges met during seeding of motile or different-sized bacteria.

$P$. amylolyticus and $X$. retroflexus are soil isolates known to engage in a dynamic multispecies biofilm with strong synergistic effects when grown in a static four-species community alongside Microbacterium oxydans and Stenotrophomonas rhizophila [14]. The recently isolated and characterized wrinkled variant of $X$. retroflexus, with enhanced biofilm formation, has shown augmented mutualistic behaviour when forming dual-species biofilms with $P$. amylolyticus and also a changed spatial organization compared to that of co-cultures containing the ancestral variant [15]. However, as $P$. amylolyticus is considerably larger than wrinkled $X$. retroflexus, correct seeding of this combination of strains and other similar ones can prove challenging (Figure 2A-D). By increasing the media viscosity and adding stain (Nigrosin) to the inoculum, we were able to successfully introduce this co-culture and establish a dual-species biofilm in a consistent highthroughput manner in the BioFlux system (Figure 3). This procedure is directly applicable to future studies of different combinations of bacterial species, enabling studies of mixed-species biofilms under continuous flow.

$P$. putida and $X$. retroflexus were used to analyze the improved BioFlux seeding procedure, based on addition of stain and enhanced viscosity, under high-throughput conditions (Figure 4). Despite the fact that the bacterial inoculum was introduced into the center of the viewing chamber, control channels at normal viscosity had in some cases upstream bacterial growth after incubation (Figure 4D-F). This was completely avoided by increasing the viscosity during seeding (Figure 4A-C). Moreover, measurements of the distance from the beginning of the biofilm formation and to the downstream edge of the viewing chamber showed improved restriction of the growth to a well-defined area of the channels and reduction of the standard deviation $(n=6)$ from $1368 \mu \mathrm{m}$ to $101 \mu \mathrm{m}$ (Figure 5).

The bacterial viability was unchanged following incubation in glycerol (Supplementary Figure 1) and the Nigrosin stain disappeared quickly upon initiating the fluidic flow, indicating that this and glycerol are removed alongside the spent media. During the experiments we acquired timelapse videos of the biofilm formation and did not observe any obvious side effects on biofilm development by using glycerol and Nigrosin for seeding (data not shown). Z-stack images of successfully established 
mixed-species biofilms also indicated that this protocol affects neither single-nor dualspecies $P$. putida and $X$. retroflexus biofilms (Figure 6).

Even though studies of bacterial growth under continuous flow can be challenging, fluid shear has great impact on the phenotype and is relevant for understanding biofilm development in natural habitats [3,5]. Mixed-species communities have traditionally not received much attention, but a recent acknowledgement of microbial communities being heterogeneous, coordinated and affected by social interactions have emphasized the importance of community-intrinsic properties [16]. With this new protocol (found in the supplementary material) we aim to enable improved understanding of unique properties emerging in communities exposed to flow and reduce the gap between studies of mono- and mixedspecies communities.

\section{BACTERIAL STRAINS \& CONDITIONS}

$\mathrm{P}_{\mathrm{A} 10403}$ gfpmut3-Gen ${ }^{\mathrm{R}}$ was introduced into P. putida KT2442 by a mini-Tn7 system previously described by Choi and Schweizer [17]. P. amylolyticus and $X$. retroflexus were originally isolated simultaneously from the same specific soil environment [18] and characterization of wrinkled variant as well as introduction of fluorescent markers were performed in a previous study [15]. All Gram-negative strains were chromosomally tagged using a Tn7-based system, while the Grampositive $P$. amylolyticus harbours plasmid pCM20 encoding gfpmut3. All strains were grown in premixed Tryptic Soy Broth (TSB) (Sigma-Aldrich). In order to prevent plasmid loss, single-species cultures of $P$. amylolyticus and dual-species cultures of $P$. amylolyticus and $X$. retroflexus were supplemented with $5 \mu \mathrm{g} / \mathrm{ml}$ erythromycin. All overnight cultures were grown at 250 R.P.M. at $24^{\circ} \mathrm{C}$.

\section{VIABILITY IN HIGH VISCOSITY}

Overnight cultures of $P$. amylolyticus, $P$. putida and $X$. retroflexus were diluted equally in either glycerol (final concentration $50 \%$ ) or PBS. In order to mimic the protocol setup, cells were incubated at room temperature for 45-60 $\mathrm{min}$ and were subsequently quantified by CFU counting on solidified TSB (1.5\% agar).

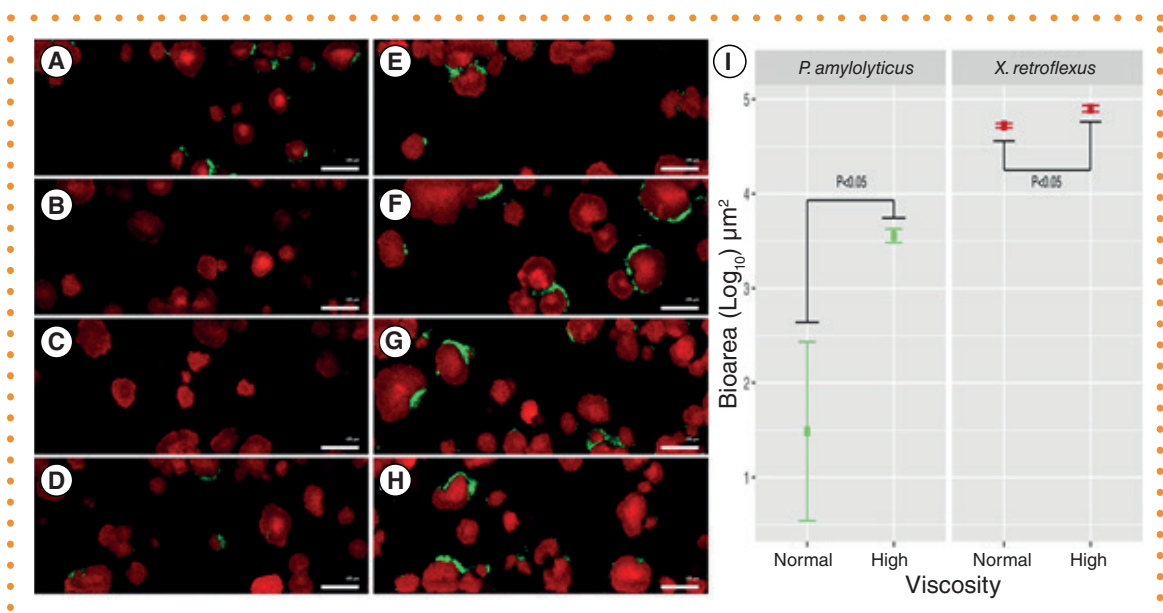

Figure 2. Consistent mixed-species biofilms when seeding at high viscosity. Representative confocal laser scanning microscopy tile regions of biofilm formation by $P$. amylolyticus (GFP, green) and a wrinkled variant of $X$. retroflexus (mCherry, red) $18 \mathrm{~h}$ post seeding with scale bars representing $100 \mu \mathrm{m}$. Seeding performed at normal viscosity (A-D) showed inferior establishment of mixedspecies biofilms compared with seeding at increased viscosity $(E-H)$. (I) Quantitative analysis indicated that the amount $P$. amylolyticus was more consistent when seeding at high viscosity and that the general biomass was increased overall (Mann-Whitney $U$ test; $n=6$, error bars represent standard error.
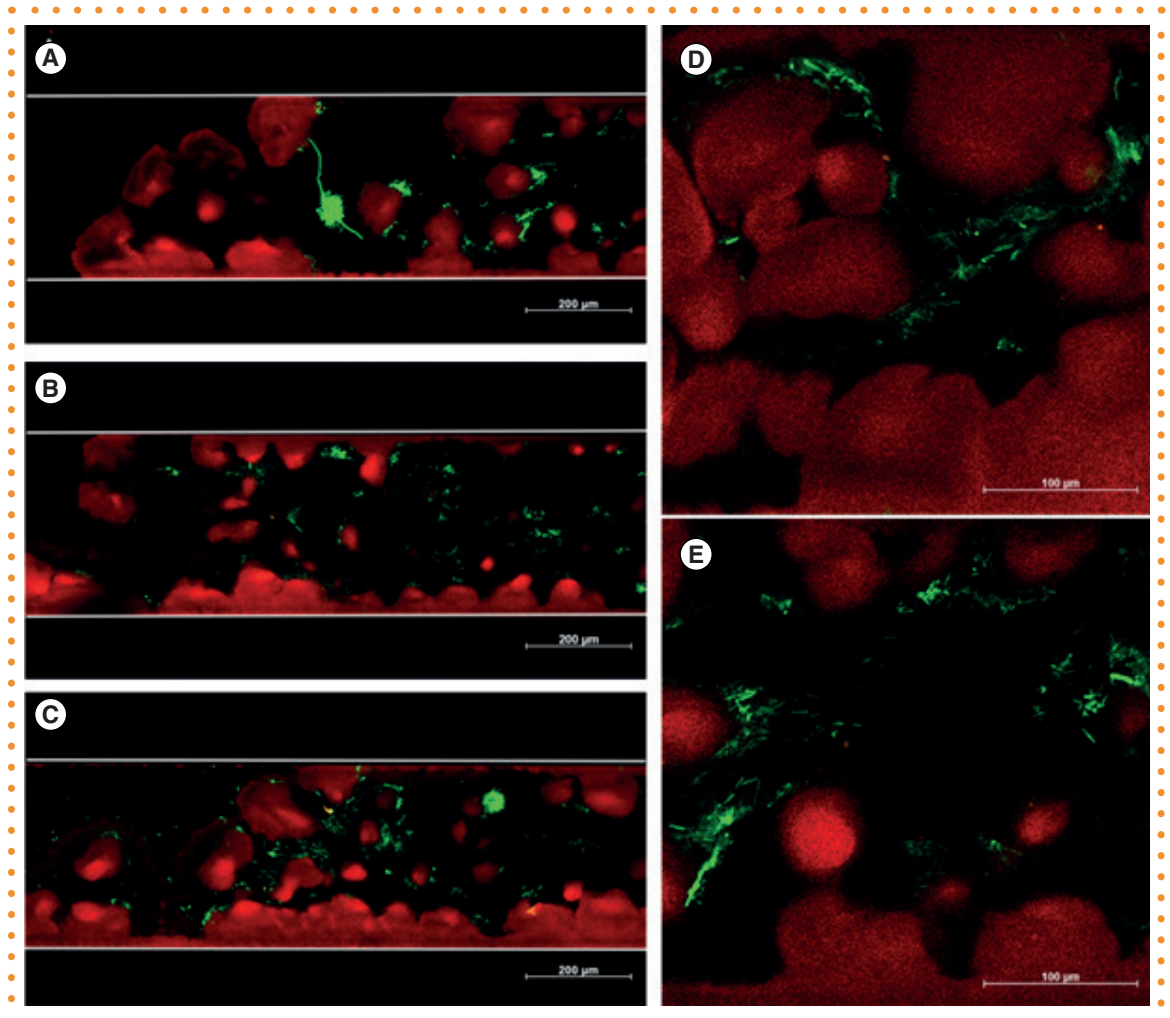

Figure 3. High-throughput dual-species biofilm formation with bacteria of different sizes at high viscosity. Representative confocal laser scanning microscopy images of $P$. amylolyticus (GFP, green) and a wrinkled variant of $X$. retroflexus (mCherry, red) $20 \mathrm{~h}$ post seeding. Five out of six channels in a column is represented as tile regions, with scale bars representing $200 \mu \mathrm{m}(A-C)$ or as single snaps, with scale bar representing $100 \mu \mathrm{m}(\mathrm{D}-\mathrm{E})$. White lines represent the side walls of the viewing chamber. 


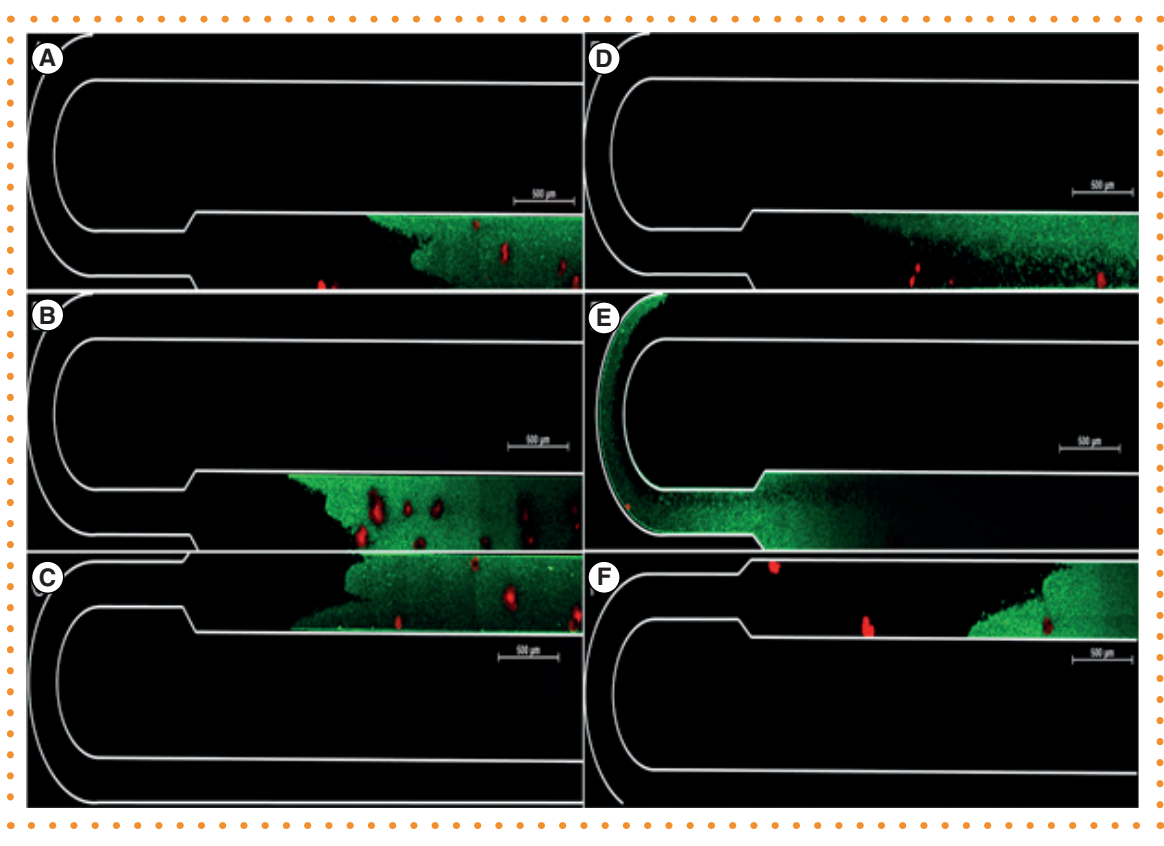

Figure 4. Consistent high-throughput multispecies biofilm formation after seeding at high viscosity. Representative confocal laser scanning microscopy images of biofilm formation by $P$. putida (GFP, green) and a wrinkled variant of $X$. retroflexus (mCherry, red) 18-h post seeding. Biofilms formed in channels from a column ( 6 channels) with increased viscosity while seeding $(A-C)$ were more consistent and well defined than those in channels from a column seeded at normal viscosity (D-F). Bacterial growth upstream of the seeding area was observed in some channels seeded at normal viscosity (illustrated in E); this was not observed in any channels seeded at high viscosity. Scale bars represent $500 \mu \mathrm{m}$.

\section{BIOFILM CULTIVATION IN THE BIOFLUX MICROFLUIDIC FLOW SYSTEM}

$X$. retroflexus and $P$. putida were tested as single- and dual-species biofilms by adjusting overnight cultures to $\mathrm{OD}_{600}=0.3$ followed by addition of glycerol (final concentration 27.5\%) or fresh medium (control) and Nigrosin (Sigma-Aldrich) to create working cultures with $\mathrm{OD}_{600}=0.15$. A reverse flow at 4 dyn $\mathrm{cm}^{-2}$ was provided until Nigrosin was visible in the viewing chamber of a 48-well plate in the BioFlux 1000 device. Subsequently, the inoculum was incubated for 15 min without flow in order to attach, before the inlet well was filled and the flow initiated at 0.15 dyn $\mathrm{cm}^{-2}$ for an $18-\mathrm{h}$ period. A

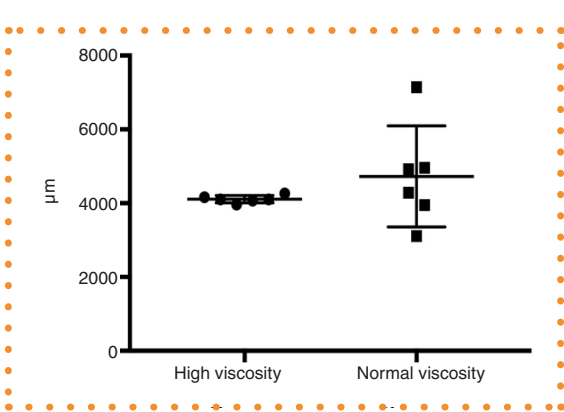

Observer Microscope Z1 (Carl Zeiss Inc.) equipped with a 10X objective.

Time-lapse videos and final image acquisition of biofilm formation were monitored and analyzed using a confocal laser scanning microscopy instrument (Zeiss LSM 800, Carl Zeiss Inc.) equipped with a $20 \mathrm{X}$ objective. Images were acquired with a 20-min interval with excitation wavelengths at 561 and $488 \mathrm{~nm}$ applied for mCherry and GFP, respectively, while maximum emission wavelengths for mCherry and GFP were 617 and $506 \mathrm{~nm}$, respectively.

Image preparation, loading and quantification of biomass volume $\left(\mu \mathrm{m}^{3}\right)$ and area $\left(\mu \mathrm{m}^{2}\right)$ were analyzed as described previously [19], using the free statistic software $\mathrm{R}[20]$.

\section{ACKNOWLEDGMENTS}

We want to thank physicist and co-founder of Fluxion Biosciences, Cristian IonescuZanetti. His theoretical and practical knowledge regarding the BioFlux microfluidic flow system was a great help in the development of this protocol. We also want to thank Nanna M. C. Olsen and Jakob Russel for their assistance with the quantitative and statistical analyses of CLSM images in $\mathrm{R}$. and $P$. amylolyticus was performed similarly, but with an increased start concentration of $P$. amylolyticus at $\mathrm{OD}_{600}=0.45$, with an attachment period lasting $30 \mathrm{~min}$ and a $20-\mathrm{h}$ incubation period. The BioFlux microfluidic flow system was located in a room cooled to $20^{\circ} \mathrm{C}$ without the heater turned on.

\section{SEEDING VERIFICATION, CONFOCAL IMAGING \& IMAGE ANALYSIS}

Priming of the channels with media, correct seeding and image acquisition of bacterial inoculum stained with Nigrosin was monitored and performed using a BioFlux $1000 Z$ system, including an Axio Inverted

Figure 5. Distance from biofilm formation to the beginning of the viewing chamber. The mean distance from the downstream beginning of the viewing chamber and to the front of the biofilm formation observed $18-h$ post seeding. Seeding at high viscosity improved delimitation of the bacterial biofilm compared to the normal viscosity seeding, restricted it to the desired area and enabled well-defined growth of biofilm ( $n=6$, error bars represent standard deviation.

\section{AUTHOR CONTRIBUTIONS}

MFH, AMT, JSM, HLR and MB were all involved in the development of study design and concept, data analysis and interpretation. gfpmut3 was introduced into P. putida by JSM. Acquisition of data was performed by MFH, AMT and HLR. Initial draft of the manuscript was written by MFH, and all authors contributed to revisions.

\section{FINANCIAL \& COMPETING INTERESTS DISCLOSURE}

This project was funded by the Villum Foundation (Young Investigator Programme, project no. 10098) and the Novo Nordisk Foundation (Grant No. NNF170C0027620). The authors have no other relevant affiliations or financial involvement with any organization or entity with a financial interest in or financial conflict with the subject matter or materials discussed in the manuscript apart from those disclosed.

No writing assistance was utilized in the production of this manuscript. 


\section{OPEN ACCESS}

This work is licensed under the AttributionNonCommercial-NoDerivatives 4.0 Unported License. To view a copy of this license, visit http://creativecommons.org/licenses/ by-nc-nd/4.0/

\section{SUPPLEMENTARY DATA}

To view the supplementary data that accompany this paper please visit the journal website at: www.future-science. com/doi/suppl/10.2144/btn-2018-0150

\section{REFERENCES}

1. Stoodley P, Sauer K, Davies DG, Costerton JW. Biofilms as complex differentiated communities. Annu. Rev. Microbiol. 56, 187-209 (2002).

2. Flemming $\mathrm{H}-\mathrm{C}$, Wingender J. The biofilm matrix. Nat. Rev Microbiol. 8(9), 623-633 (2010).

3. Hall-Stoodley L, Costerton JW, Stoodley P. Bacterial biofilms: from the natural environment to infectious diseases. Nat Rev. Microbiol. 2(2), 95-108 (2004).

4. Burmølle M, Thomsen TR, Fazli M et al. Biofilms in chronic infections - a matter of opportunity - monospecies biofilms in multispecies infections. FEMS Immunol. Med. Microbiol. 59(3), 324-336 (2010).

5. Stoodley P, Cargo R, Rupp CJ, Wilson S, Klapper I. Biofilm material properties as related to shear-induced deformation and detachment phenomena. J. Ind. Microbiol. Biotechnol. 29(6), 361-367 (2002).

6. Lee KWK, Periasamy S, Mukherjee M, Xie C, Kjelleberg S, Rice SA. Biofilm development and enhanced stress resistance of a model, mixed-species community biofilm. ISME 8(4), 894-907 (2014).

7. Benoit MR, Conant CG, lonescu-Zanetti C, Schwartz M, Matin A. New device for high-throughput viability screenin of flow biofilms. Appl. Environ. Microbiol. [Internet]. 76(13), 4136-4142 (2010).

8. Ramsey MM, Whiteley M. Pseudomonas aeruginosa attachment and biofilm development in dynamic environments. Mol. Microbiol. 53(4), 1075-1087 (2004).

9. Azeredo J, Azevedo NF, Briandet R et al. Critical review on biofilm methods. Crit. Rev. Microbiol. 43(3), 313-351 (2017)

10. Burmølle M, Ren D, Bjarnsholt T, Sørensen SJ. Interactions in multispecies biofilms: do they actually matter? Trends Microbiol. 22(2), 84-91 (2014)

11. McClaine JW, Ford RM. Reversal of flagellar rotation is important in initial attachment of Escherichia coli to glass in a dynamic system with high- and low-ionic-strength buffers. Appl. Environ. Microbiol. 68(3), 1280-1289 (2002).

12. Cheng $\mathrm{N}-\mathrm{S}$. Formula for the viscosity of a glycerol-water mixture. Ind. Eng. Chem. Res. 47(9), 3285-3288 (2008).

13. Volk A, Kähler CJ. Density model for aqueous glycerol solutions. Exp. Fluids. 59(5), 75 (2018).

14. Ren D, Madsen JS, Sørensen SJ, Burmølle M. High prevalence of biofilm synergy among bacterial soil isolates in cocultures indicates bacterial interspecific cooperation. ISME J. 9(1), 81-89 (2015).

15. Røder HL, Herschend J, Russel J et al. Enhanced bacterial mutualism through an evolved biofilm phenotype. ISMEJ. 12(11), 2608-2618 (2018).

16. Madsen JS, Sørensen SJ, Burmølle M. Bacterial social interactions and the emergence of community-intrinsic properties. Curr. Opin. Microbiol. 42, 104-109 (2018).

17. Choi K-H, Schweizer HP. mini-Tn7 insertion in bacteria with single attTn7 sites: example Pseudomonas aeruginosa. Nat Protoc. 1(1), 153-161 (2006).

18. Ren D, Madsen JS, de la Cruz-Perera $\mathrm{Cl}$, Bergmark $\mathrm{L}$, Sørensen SJ, Burmølle M. High-throughput screening of multispecies biofilm formation and quantitative PCR-base assessment of individual species proportions, useful for exploring interspecific bacterial interactions. Microb. Ecol. 68(1), 146-154 (2014).

19. Liu W, Russel J, Røder HL, Madsen JS, Burmølle M, Sørensen SJ. Low-abundant species facilitates specific spatial organization that promotes multispecies biofilm formation organization that promotes multispecies bio
Environ. Microbiol. 19(7), 2893-2905 (2017).

20. $R$ Core Team. $R$ : a language and environment for statistical computing. R Foundation for Statistical Computing, Vienna. 201 (2018). www.R-project.org
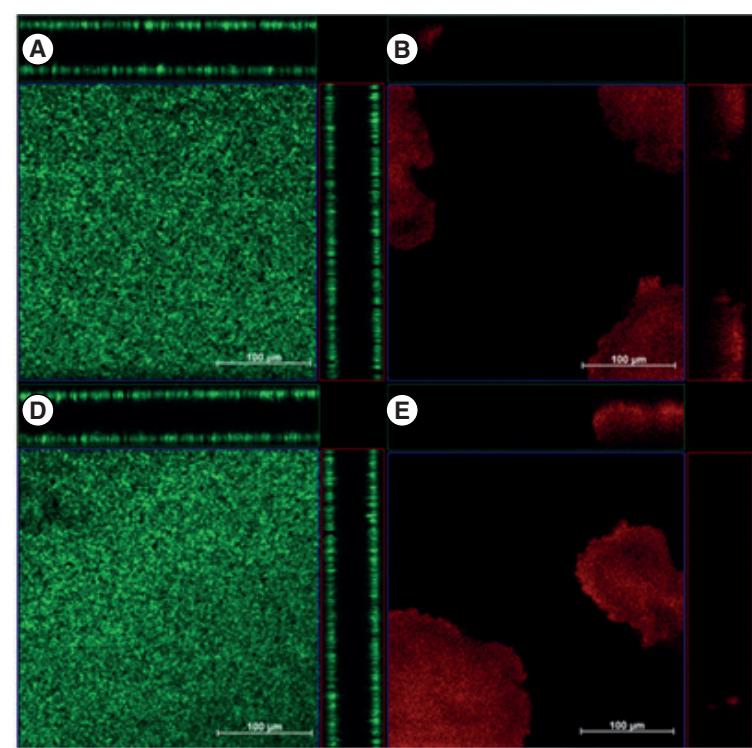

c
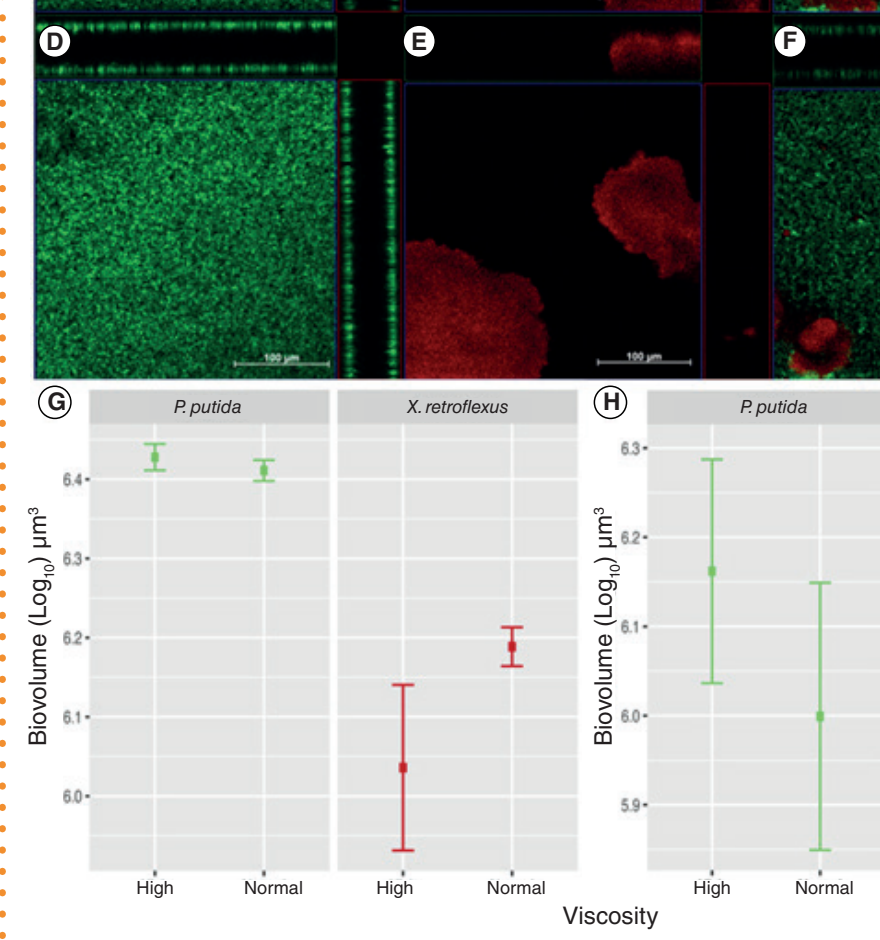

E
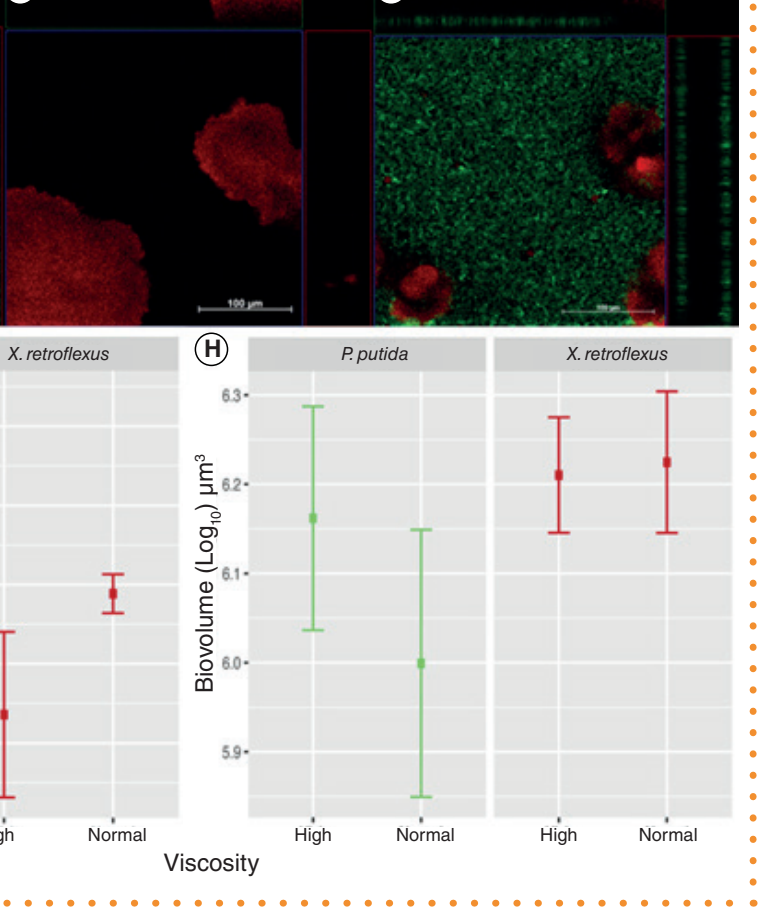

Figure 6. Comparison of z-stack images of successful biofilm formation at normal and high viscosity. Representative confocal laser scanning microscopy z-stack images of successfully established single- and dual-species biofilms at normal and high viscosity of $P$. putida (GFP, green) and wrinkled $X$. retroflexus (mCherry, red) 18-h post seeding. Scale bars represent $100 \mu \mathrm{m}$. High viscosity medium was used for seeding in the top row images $(A-C)$, and the final biofilm formation looked similar to when seeding was successfully performed at normal viscosity $(D-F)$, indicating that seeding under improved conditions does not affect final biofilm formation. This was supported by quantitative analysis of the biomass volume $\left(\mu \mathrm{m}^{3}\right)$ of the single species- $(G)$ and mixed-species biofilms $(H)(n=3$, error bars represent standard error. 